\title{
Microdissected Prefabricated Flap: An Evolution in Flap Prefabrication
}

\author{
Süleyman Taş \\ Department of Plastic, Reconstructive and Aesthetic Surgery, Medical Park Hospital, Istanbul, Turkey
}

\begin{abstract}
When traditional flap techniques are not feasible, we apply flap prefabrication, which is more complicated and sophisticated but supplies large and thin flaps. There are some disadvantages to the technique that require improvement, such as venous congestion after flap transfer, which requires months for neoangiogenesis and necessitates a vascular carrier. Here, the author presents a new technique, called as 'microdissected prefabricated flap', to successfully produce a safe, large, and thin flap. This technique is based on the microdissection of the perforators to the greatest extent possible, spreading them out into the subdermal level and using them as a carrier. The details and the application of this technique are presented and reported.
\end{abstract}

Keywords Fluorescein / Injections / Surgical flap / Microdissection / Microsurgery

\begin{abstract}
Correspondence: Süleyman Taş Department of Plastic, Reconstructive and Aesthetic Surgery, Medical Park Hospital, Gaziosmanpasa, Istanbul, 34245 , Turkey

Tel: $+90-532-0563693$

Fax: $+90-212-9793000$

E-mail: drsuleymantas@live.com

This article contains supplemental Video S1 and Slide 1.

This report was presented as an extended oral presentation at the 7th European Plastic Surgery Research Council (EPSRC) Congress, 27-30 August 2015, in Hamburg, Germany, and the abstract of the presentation was published in Plastic and Reconstructive Surgery (Taş S. A new concept in flap prefabrication by supermicrosurgery: microdissected prefabricated flap. Plast Reconstr Surg 2015;136:437-8).
\end{abstract}

No potential conflict of interest relevant to this article was reported.

\section{INTRODUCTION}

Flap prefabrication refers to the production of an axial pattern flap via the implantation of a vascular carrier into the subdermal level [1]. It consists of two parts: the selection of a donor site and the creation of a vascular carrier. In case of massive facial resurfacing, cervicothoracic skin is more appropriate as a donor site because of the color and texture match. An ideal vascular carrier should have large-caliber blood vessels with long pedicles for microvascular anastomosis, and it should not include any tissue other than a dense capillary network [2]. In the literature, muscle, fascia [3-7], omentum [8], and vessel bundles [9-11] have all been applied as the vascular carrier.

On the other hand, the author has attempted to use the perforator vessels in the donor site as a carrier by microdissecting the perforators until it is possible to obtain the main pedicle with a connection to the subdermal plexus. The author has tried to eliminate the disadvantages of the classic prefabricated flap technique. This procedure is classified as a 'microdissected prefabricated flap.' The technique presented here was used for 2 patients on the abdominal area, where it is a challenge to obtain a safe, large and thin flap due to the anatomic difference in the adipose 
layers of the abdomen.

\section{IDEA}

The details of the technique presented here and a comparison with the classic prefabricated flap technique are demonstrated in the Video Animation (Supplemental Video S1). Following the marking of the perforators, the planning of the flap and expander pouch design, the first step in the operative procedure is to elevate the flap classically as a deep inferior epigastric perforator (DIEP) flap. Deep adipose tissue should be removed sharply from the perforators beneath Scarpa's fascia. However, above Scarpa's fascia, there is an extensive branching of the perforators, which often split into five or more branches at this level and then anastomose with the 'subdermal plexus' beneath the dermis $[12$, 13]. Therefore, the second step should be microdissection, which was first described by Kimura et al. [14,15]. They used this technique for a thin tensor fascia lata and anterior thigh flap. At the start of microdissection, Scarpa's fascia should be removed sharply around the perforator using microscissors. Continuing into Camper's fascia, adipose tissue around the perforator can be removed bluntly using microforceps up to the subdermal plexus. Because of the branching at this level, the largest branch of the perforator or as many branches of perforators as possible should be protected during the microdissection. The vascular supply can be followed with the bleeding of the edges of the flap (Fig. 1).

The flap thickness is decreased from 30 to $4 \mathrm{~mm}$ after microdissection. To ensure a vascular supply from the perforator network, a $2 \mathrm{~mL}$ intra-arterial fluorescein sodium injection can be performed via the DIEP artery with a $33 \mathrm{G}$ needle. After the injection, partial yellow staining on the skin is inspected and a shimmering appearance should be observed using a Wood's lamp (Fig. 2). Although there will be a partial yellow staining on the skin, the flap circulation is good and this shows that the supply from both subcutaneous and microdissected perforators are important for the viability of the presented flap, demonstrating the necessity of prefabrication. Because microdissection leads to relative elongation of the pedicle length, we obtained a skeletonized vascular network continuous with the subdermal plexus. Then, this network is spread out beneath the designed flap by placing several transcutaneous sutures around the side branches. Finally, a slightly inflated tissue expander is placed under the flap (Supplemental Slide 1).

\section{Case 1}

A 48-year-old man was admitted with a large skin defect on his anal and external genital area secondary to Fournier's gangrene. He underwent one graft and one flap surgery at another center, but these procedures failed due to infection. The result of the culture was resistant Pseudomonas infection and skin flora. Below the umbilicus, a $14 \mathrm{~cm} \times 14 \mathrm{~cm}$ area was microdissected and prefabricated on right DIEP vessels. At 5 weeks, the microdissected prefabricated abdominal flap, $14 \mathrm{~cm} \times 7 \mathrm{~cm}$ in size, was transferred as a pedicle flap for coverage of the anoscrotal area. The donor site was closed with the remaining $14 \mathrm{~cm} \times 7 \mathrm{~cm}$ flap and healed without complications. At 6 months' follow-up, the result was satisfactory (Fig. 3).

\section{Fig. 1. Operative technique}

(A) Following completion of the microdissection, achieving the vascular network, flap thickness, and vascular supply can be observed. Bleeding from the flap margins (white arrows) indicates good vascular supplementation despite thinning. (B) The skeletonized vascular network was spread out beneath the flap and a tissue expander was inserted beneath the flap. With the technique presented here, the flap thickness was reduced from 30 to $4 \mathrm{~mm}$.
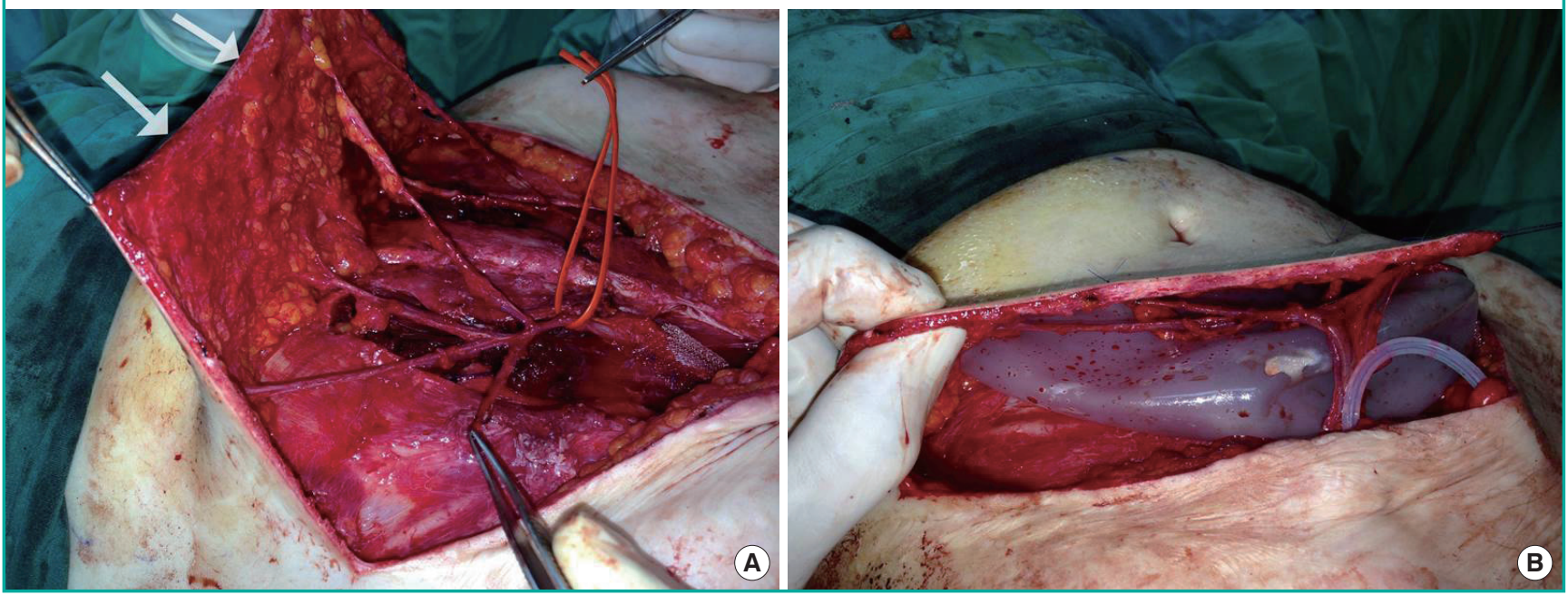


\section{Case 2}

A 22-year-old female patient presented with a severe burn injury dating from when she was 2 years old. The patient underwent 10 procedures at other institutions. However, she still presented a serious facial disfigurement and dysfunctions of the mouth, eyelid, ear and neck. All possible donor sites for free tissue transfer had been utilized and computed tomography angiography showed that there were no recipient vessels to serve as a facial artery or superficial temporal artery for anastomosis up to the neck. Below the umbilicus, a $18 \mathrm{~cm} \times 7 \mathrm{~cm}$ scarless area was chosen for the presented technique, and this area was prefabricated on bilateral DIEP vessels. Two months after flap prefabrication, the facial scars were excised and the microdissected prefabricated abdominal flap, $30 \mathrm{~cm} \times 15 \mathrm{~cm}$ in size, was transferred as a free flap via anastomosing to bilateral superior thyroid vessels for coverage of the upper two-thirds of the face. The donor site was primarily closed and healed without complications. Five days after flap transfer, the flap was revised to form the eyelids. One month later, the patient underwent a Z-plasty procedure for cervical and lower lip contracture and reshaping of the nose and ear. At 12 months' follow-up, the patient could see without difficulty, breathe, and was pleased with the cosmetic result (Fig. 4, Supplemental Slide 1).

\section{Fig. 2. Intra-arterial fluorescein sodium injection}

To ensure the vascular supply from the perforator network, $2 \mathrm{~mL}$ of intra-arterial fluorescein sodium was injected via the deep inferior epigastric perforator artery with a $33 \mathrm{G}$ needle. After the injection, (A) yellow staining on the skin was inspected and (B) a shimmering appearance was observed using a Wood's lamp.
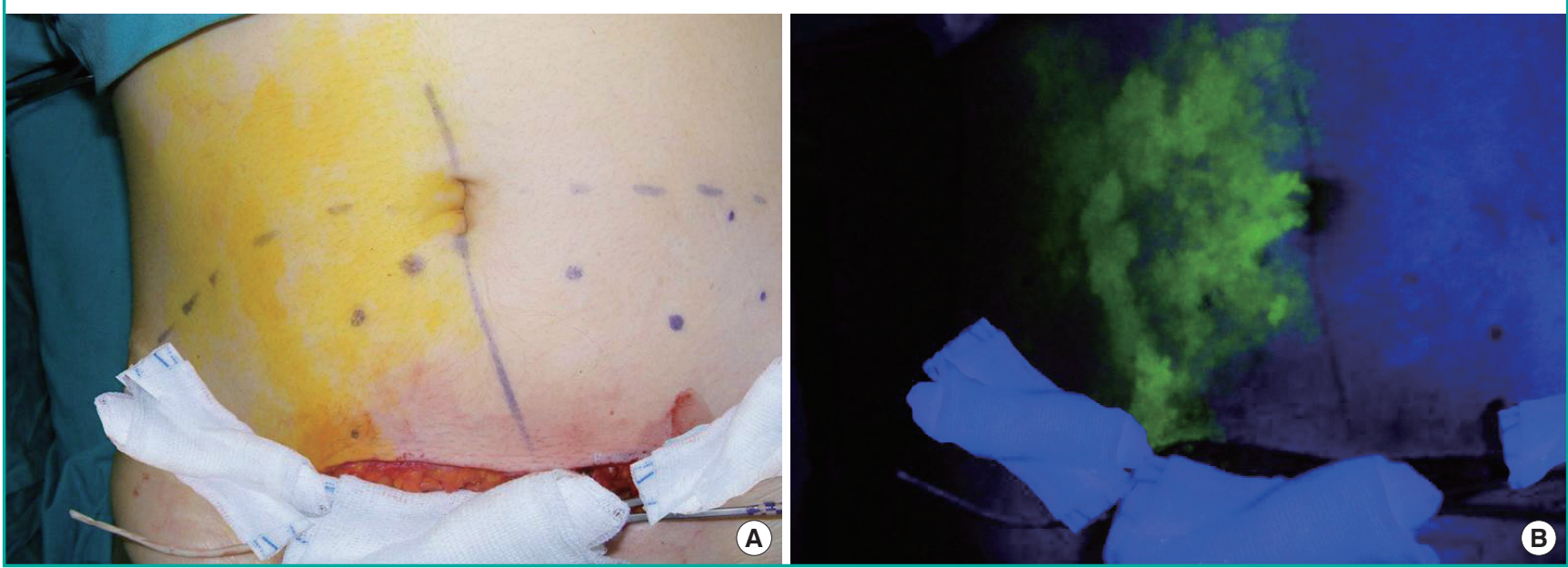

Fig. 3. A pedicle flap example

Case 1. (A) Preoperative.

(B) 6 months' postoperatively.
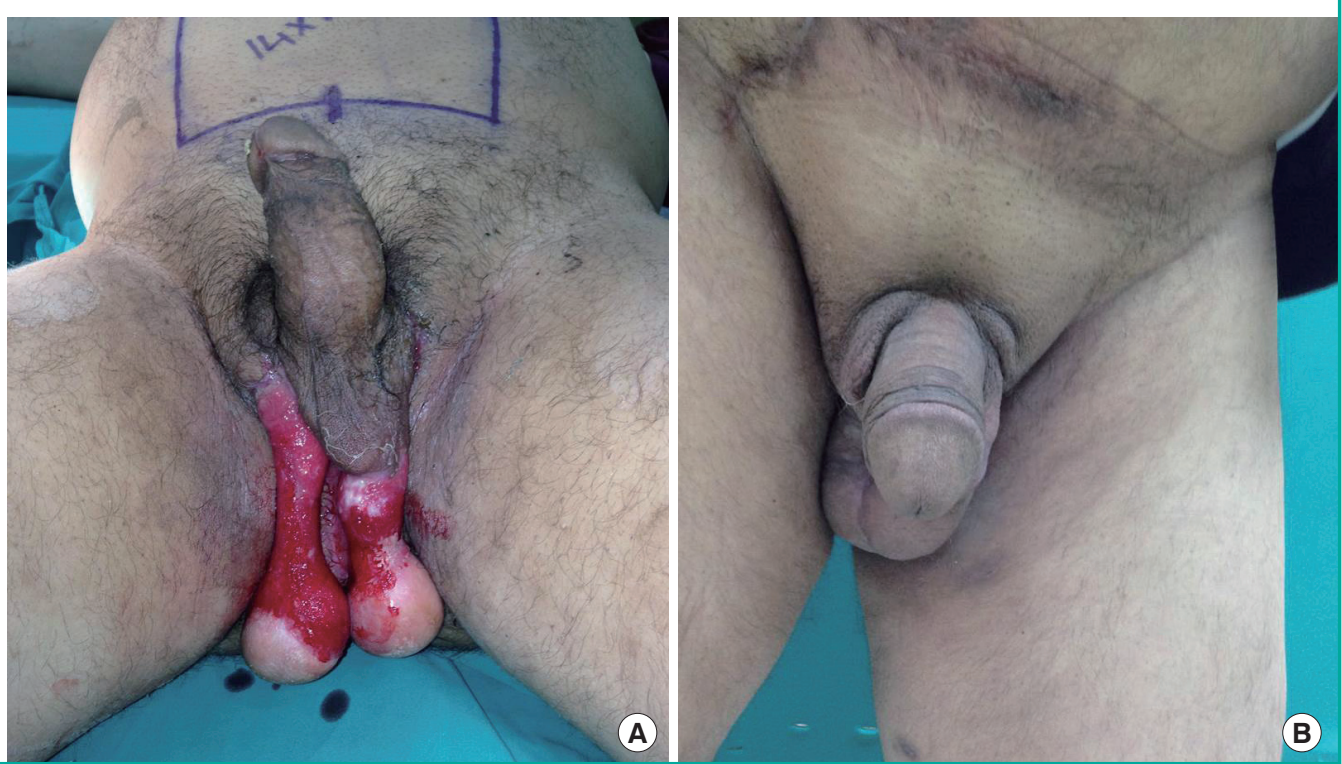
Fig. 4. A free flap example

Case 2. (A) Preoperative.

(B) 12 months' postoperatively.
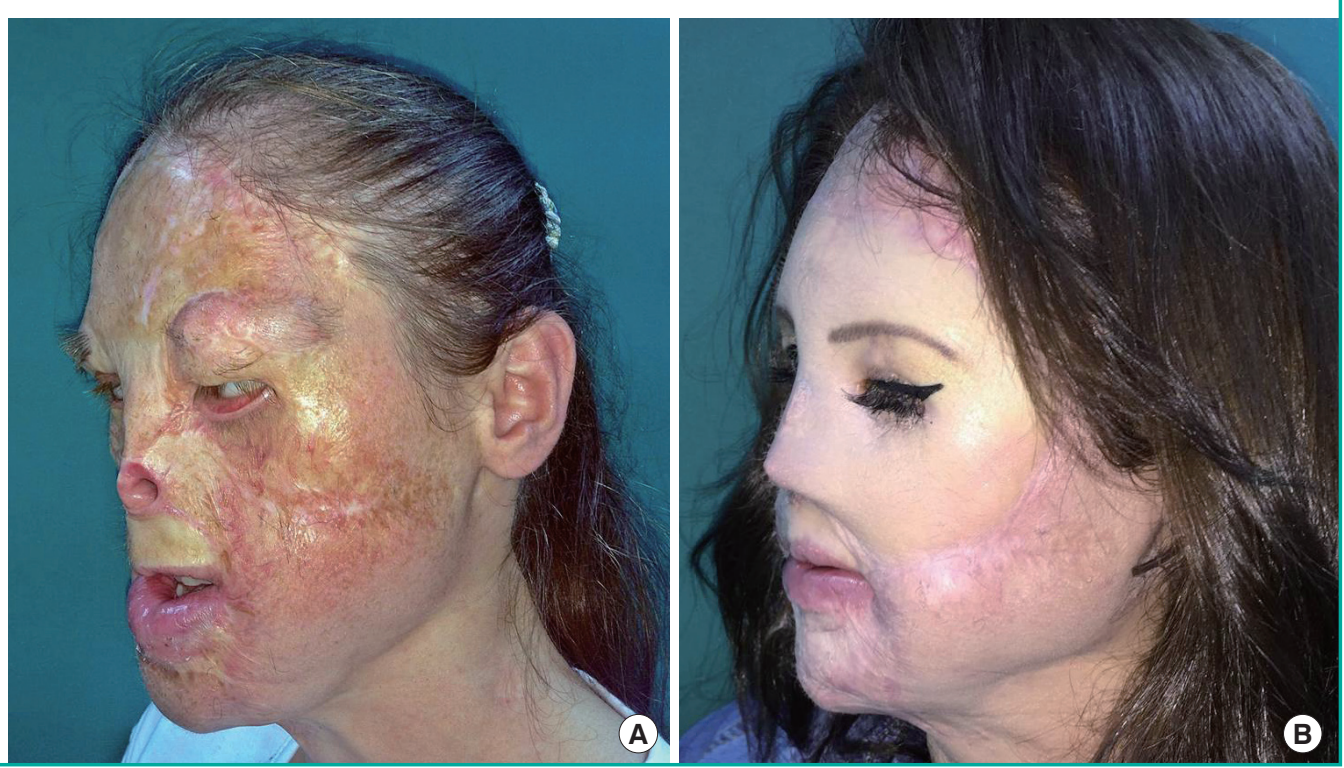

\section{DISCUSSION}

Classically, thinning of DIEP flap can only be performed safely under Scarpa's fascia. Otherwise, thinning over Scarpa's fascia threatens the blood supply of the flap [13]. Reported superthin DIEP flaps have a $10-12 \mathrm{~mm}$ thickness [12,13]. However, a considerable reduction of thickness was achieved, up to $4 \mathrm{~mm}$, with the new technique presented here. Since extensive fat tissue excision is performed with the microdissection technique, vascular supplements from the perforator vessels might be weakened. However, because the subdermal vascular plexus remains intact, the flap also supplies a random-pattern flap and flap circulation does not break down. To ensure the vascular supply from the perforator network, intra-arterial fluorescein sodium can be injected as described here. Intravenous injection of fluorescein sodium is a well-known tool for supporting flap survival; however, to the best of our knowledge, intra-arterial fluorescein sodium injection has not been reported before.

The time of maximal neovascularization following pedicle implantation has not yet been indicated in humans. Experimental studies in rabbits and rats show that it occurs quickly, within 2 or 3 weeks, but in humans it takes at least 8 weeks [3]. In reported cases in the literature concerning large defects, the flaps were transferred 6 months after prefabrication $[2,7]$. This is a considerably long time. In the presented report, the prefabricated flaps were transferred in 5 and 8 weeks, respectively.

On the other hand, venous congestion is a common problem following the transfer of a prefabricated flap and it may cause marginal necrosis. Pribaz and Guo [1] showed that the reason for venous congestion may be unequal neovascularization due to changes in the pressure of the vessels. Solutions to this problem have been attempted with the delay phenomenon, leeching, prolonging prefabrication, and additional venous anastomosis. However, these have not been sufficient, and the ratio of the size between the implanted fascia flap and the prefabricated flap may play an important role in preventing venous congestion, though an exact ratio has not yet been reported [7]. Lastly, a carrier is required to create an axial pattern flap in the classic prefabrication technique, which means additional donor side morbidity.

Despite these problems, in the presented technique, because the implanted vascular network has continued with a subdermal plexus, it provides fast vessel maturation and does not lead to complications such as venous congestion and partial necrosis, nor does it require a vascular carrier, preventing donor site morbidity.

In conclusion, a 'microdissected prefabricated flap' could be a reliable alternative tool for the resurfacing of massive soft tissue. However, experimental studies should be conducted to determine how we can derive maximum benefit from this technique. Finally, regarding the problem of achieving a tissue complex as total face transplantation, the author believes that it may be possible with this technique.

\section{ORCID}

Süleyman Taş http://orcid.org/0000-0001-8874-3009

\section{REFERENCES}

1. Pribaz JJ, Guo L. Flap prefabrication and prelamination in 
head and neck reconstruction. Semin Plast Surg 2003;17: 351-62.

2. Li Q, Zan T, Li H, et al. Flap prefabrication and stem cell-assisted tissue expansion: how we acquire a monoblock flap for full face resurfacing. J Craniofac Surg 2014;25:21-5.

3. Pribaz JJ, Fine N, Orgill DP. Flap prefabrication in the head and neck: a 10-year experience. Plast Reconstr Surg 1999; 103:808-20.

4. Khouri RK, Ozbek MR, Hruza GJ, et al. Facial reconstruction with prefabricated induced expanded (PIE) supraclavicular skin flaps. Plast Reconstr Surg 1995;95:1007-15.

5. Teot L, Cherenfant E, Otman S, et al. Prefabricated vascularised supraclavicular flaps for face resurfacing after postburns scarring. Lancet 2000;355:1695-6.

6. Kimura N, Hasumi T, Satoh K. Prefabricated thin flap using the transversalis fascia as a carrier. Plast Reconstr Surg 2001; 108:1972-80.

7. Li Q, Zan T, Gu B, et al. Face resurfacing using a cervicothoracic skin flap prefabricated by lateral thigh fascial flap and tissue expander. Microsurgery 2009;29:515-23.

8. Erol OO, Spira M. Reconstructing the breast mound employing a secondary island omental skin flap. Plast Reconstr
Surg 1990;86:510-8.

9. Morrison WA, Penington AJ, Kumta SK, et al. Clinical applications and technical limitations of prefabricated flaps. Plast Reconstr Surg 1997;99:378-85.

10. Yao ST. Vascular implantation into skin flap: experimental study and clinical application: a preliminary report. Plast Reconstr Surg 1981;68:404-10.

11. Margulis A, Agam K, Icekson M, et al. The expanded supraclavicular flap, prefabricated with thoracoacromial vessels, for reconstruction of postburn anterior cervical contractures. Plast Reconstr Surg 2007;119:2072-7.

12. Rozen WM, Murray AC, Ashton MW, et al. The cutaneous course of deep inferior epigastric perforators: implications for flap thinning. J Plast Reconstr Aesthet Surg 2009;62:98690.

13. Prasetyono TO, Bangun K, Buchari FB, et al. Practical considerations for perforator flap thinning procedures revisited. Arch Plast Surg 2014;41:693-701.

14. Kimura N. A microdissected thin tensor fasciae latae perforator flap. Plast Reconstr Surg 2002;109:69-77.

15. Kimura N, Satoh K, Hosaka Y. Microdissected thin perforator flaps: 46 cases. Plast Reconstr Surg 2003;112:1875-85.

Supplemental Video S1. The details of technique presented here and comparison with the classic prefabricated flap technique are demonstrated as a video animation, which can be seen at the following link, https://youtu.be/6vdp45G8PCM.

\section{Supplemental data can be found at: http://e-aps.org/src/sm/aps-43-599-s001.avi}

Supplemental Silde 1. A slideshow was prepared for other photos related to the operative technique, containing preoperative and postoperative photos of the patients in a flowchart.

Supplemental data can be found at: http://e-aps.org/src/sm/aps-43-599-s002.avi 